\title{
SMART SHOPPING CART USING RFID TECHNOLOGY
}

\author{
Susanna M Santhosh \\ Computer Science and Engineering \\ Mar Baselios Institute of Technology and Science, \\ Kothamangalam, India \\ Jills Joseph \\ Computer Science and Engineering \\ Mar Baselios Institute of Technology and Science \\ Kothamangalam, India
}

\begin{abstract}
In our project we propose an IoT based shopping system in which a Radio Frequency Identification (RFID) technology is used. Nowadays, the use of barcode for products presents several limitations, it required line of site, has low range and have less security. RFID technology identifies the product in a unique way, and provides more information than barcode. The main objective of our project is to provide a technology oriented, easily scalable system for assisting shopping inperson. Each product in the supermarket is attached with an RFID tag, the tag contains the details of the product including product id, brand name, price of the product, manufacturing date, expiry date etc. The RFID reader module is installed on the shopping trolley. When a registered user comes to the supermarket, he/she login to the corresponding trolley taken buy them using the mobile application installed on their mobile. When an item is placed in the trolley it is read automatically by the RFID reader and add it to the shopping cart. As a result billing can be done directly from the shopping cart itself.
\end{abstract}

\section{INTRODUCTION}

In this project we have worked on a solution for difficulties faced by customers in standing in a queue for billing process in a supermarket. In the last few decades technology has evolved a lot. In the era of IoT interaction between physical things comes into reality. This has bought a revolution in many sectors of the society. In our system the inventory management is made much easier so that there is no need of a laborer for items to manually scanned. In our proposed system each smart trolley is equipped with a RFID reader, micro controller and a LCD display. Micro controller is installed on the cart for data processing and LCD display is equipped as a user interface which will show the updated bill every time the shopper add or remove any object from the cart. When the customer is finished shopping bill is generated in the shopping cart and bill amount

\author{
Cemone P Babu \\ Computer Science and Engineering \\ Mar Baselios Institute of Technology and Science, \\ Kothamangalam, India \\ Preethu Benny \\ Computer Science and Engineering \\ Mar Baselios Institute of Technology and Science \\ Kothamangalam, India
}

is directly deducted from the shopping wallet provided to the user, so there is no need of staff for billing.

On behave of the RFID module several functions are performed behind the scene, including product handling, stock management, trolley management and payment. Product handling deals with product category, product details and product RFID tagging. These functions makes sure the proper management of products in the store. Stock management deals with the product quality, product quantity and minimum stock maintenance which ensures the proper management of stock in the store. In the trolley management interaction with customer and RFID module is managed effectively. In the payment module along with the billing details the customers wallet details are verified.

This work identifies the requirements for the designing of a smart shopping system and build a prototype prototype system to test functionality, and design a secure communication protocol to make the system practical. This smart shopping system is proposed with security under consideration. Our proposed system is easy to use and no special training is required.

\section{LITERATURE REVIEW}

In the paper entitled "Intelligent shopping cart" the system consists of three modules user interface and display component (UIDC), server communication component (SCC) and automatic billing component (ABC). Here RFID is used for product identification instead of using barcodes.

The paper entitled "Smart shopping trolley using RFID", the system creates an automated billing system in malls. It directly sent the product information to the billing system using ZigBee. Here every trolley is joined with a RFID reader per user and a RFID tag per user. 


\section{International Journal of Engineering Applied Sciences and Technology, 2020 \\ Vol. 5, Issue 2, ISSN No. 2455-2143, Pages 439-444 \\ Published Online June 2020 in IJEAST (http://www.ijeast.com)}

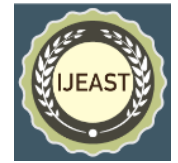

In the paper entitled "smart shopping using smart trolley", instead of using barcodes this system use rfid tags which is read by a rfid tag and a Bluetooth module is implemented in every trolley.

\section{PROPOSED SYSTEM}

RFID technology is changing into preferred technology instead of barcode system. RFID is a system with automatic identification method. In this paper we have developed a shopping cart system which use this RFID technology

In this system we have a shopping trolley which is integrated with a RFID reader module and products are tagged with RFID tags. Each shopping trolley is attached with a QR code and when a customer enters the supermarket, he/she scan the QR code and logins to the assigned trolley and start shopping. When the customer drops an item into the trolley the item is automatically read by the RFID reader and add it to the shopping cart.

When the customer is finished shopping bill is generated in the shopping cart and bill amount is directly deducted from the shopping wallet provided to the user, so there is no need of staff for billing.

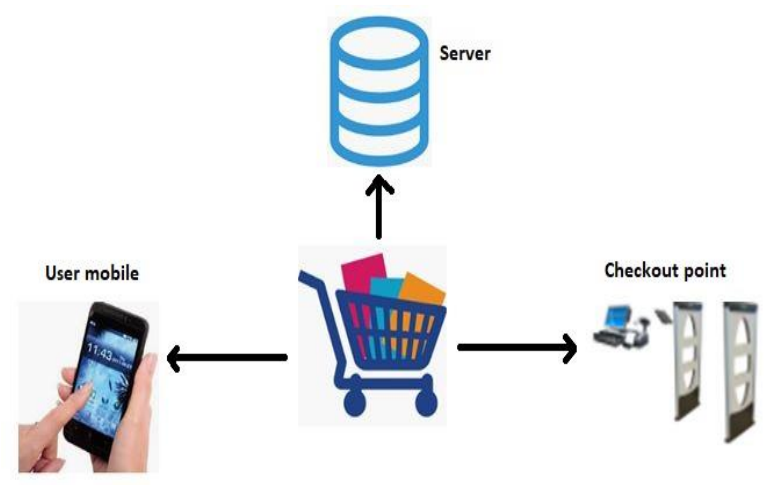

Several functions are performed behind the scene, including product handling, stock management, trolley management and payment. Product handling deals with product category, product details and product RFID tagging. These functions makes sure the proper management of products in the store. Stock management deals with the product quality, product quantity and minimum stock maintenance which ensures the proper management of stock in the store. In the trolley management interaction with customer and RFID module is managed effectively. In the payment module along with the billing details the customers wallet details are verified.

The requirements for designing of a smart shopping system are identified and build a prototype system to test functionality, and design a secure communication protocol to make the system practical. This smart shopping system is proposed with security under consideration. Our proposed system is easy to use and no special training is required.

\section{HARDWARE AND SOFTWARE IMPLEMENTATION}

\section{HARDWARE}

\section{(I) RFID Reader}

A RFID Reader is a device that uses radio-frequency waves to wirelessly track the RFID tag attached to the objects. Radio receiver, radio transponder, radio transmitter are the main parts consist. in an RFID. Electromagnetic pulse from a RFID reader that is triggered, the tag transmits the digital data.

In an RFID reader transmitter and receiver is consist as RF module. Transmitter have an oscillator. Receiver contains a demodulator. Modulator makes an impact on data commands.

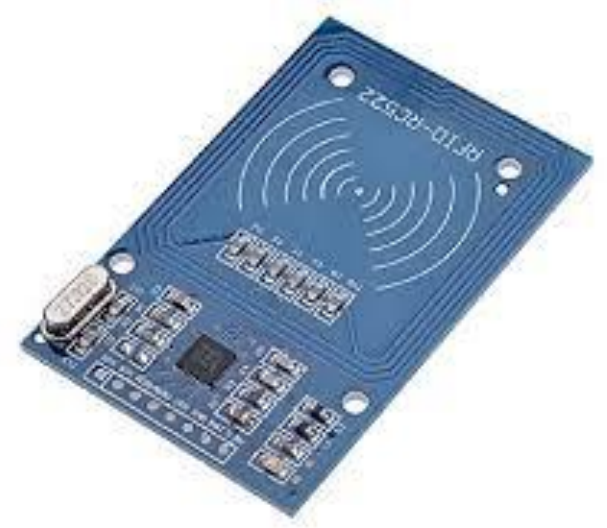

Fig: RFID Reader

\section{(II)RFID Tag}

RFID tags are of two types

Passive tag which have less battery life. They operate at a maximum distance of 3 meters. These tags have only power when it is connected with rfid readers. Simplest passive tags can hold something in a range of 64 bits of factory-written unique data and they are called "class 0 " tags.

Active tag which have battery life, have their own power source. They can transmit and process data actively and intensively over a considerable physical distance. These tags can communicate with readers 100 meters or more away.

RFID tag contain 2 parts. One is integrated circuit and other is an antenna. Integrated circuit for modulating, demodulating and storing the processing information. Antenna is for receiving and transmitting signal. 


\section{International Journal of Engineering Applied Sciences and Technology, 2020 \\ Vol. 5, Issue 2, ISSN No. 2455-2143, Pages 439-444 \\ Published Online June 2020 in IJEAST (http://www.ijeast.com)}

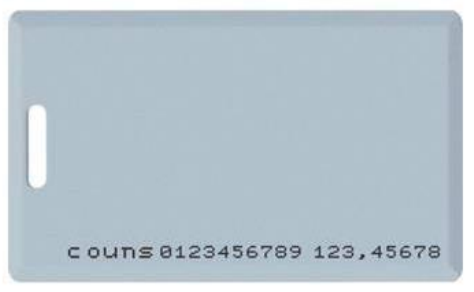

Fig: RFID Tag

\section{(III) ESP32}

It is a series of low cost, low power system on a chip microcontrollers. ESP32 is a successor o he ESP8266 microcontroller. It has a memory of $520 \mathrm{KiB}$ SRAM.

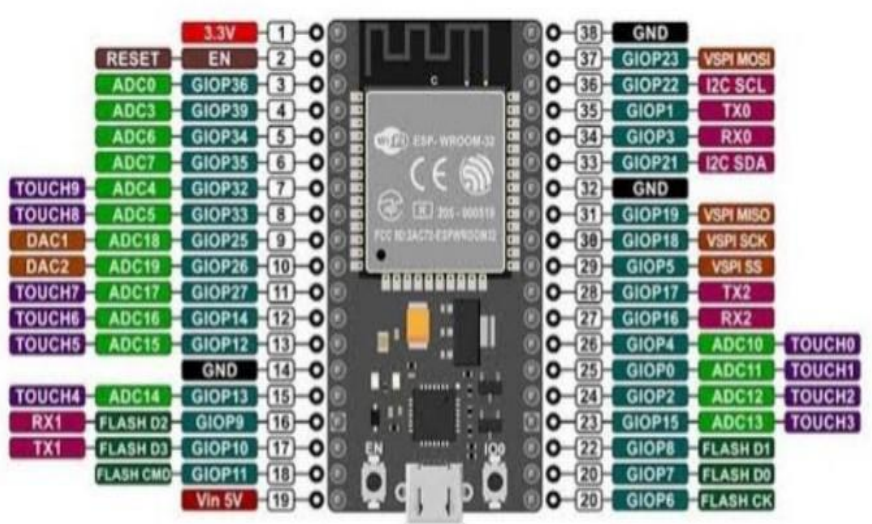

Fig: ESP32

It also includes state of the art features, such as fine grained clock gating, various power modes and dynamic power scaling. is highly integrated with in-built antenna switches, RF balum, power amplifier, filters, and power management modules.

It can perform as a standalone system or as a slave device to a host MCU. It can interface with other systems to provide $\mathrm{WiFi}$ and Bluetooth functionality through its SPI or UART interfaces

\section{(IV) OLED DISPLAY}

OLED (Organic light emitting diode) is a light emitting diode in which there is an emissive electroluminescent layer, made of from a film of organic compound that emits light in response to an electric current. OLED is also known as an organic EL diode. It is used to create digital displays in devices.

It works without a backlight because it emits visible light. OLED screen can achieve a higher contrast ratio when compared with a LCD. Benefits of OLED screen are they can be extremely thin, flexible and even rollable

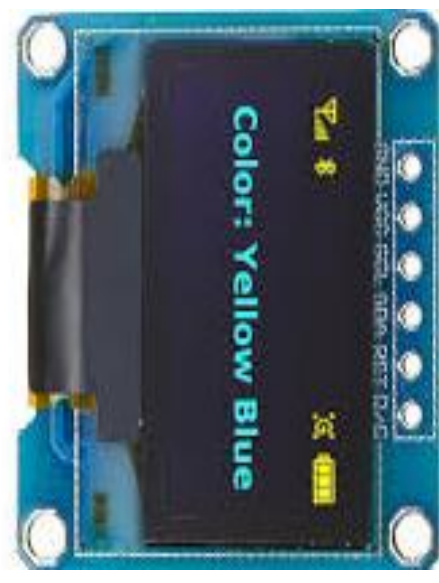

Fig: OLED display

\section{SOFTWARE}

\section{(I) VISUAL STUDIO CODE}

We used visual studio code it is a source code editor that is developed by Microsoft for windows. It is based on electron, a framework which is used to develop node. It is a development tool or building cross platform and cloud applications.

\section{(II) ANDROID STUDIO}

It is Android's official IDE. It is purpose for built Android to accelerate the development. It also help to build the highest quality apps for every devices.

\section{(III) NETBEANS}

This an integrated development environment (IDE) for java. It allow application to be developed on a set of software components.

\section{ADVANTAGES AND DISADVANTAGE}

\section{ADVANTAGES}

1. It reduce shopping time.

2. The rfid tags can be rewrite.

3. Man power at billing counters can be reduced.

4. Doesn't need line of sight.

5. Improved read rates.

6. It is trustworthy, highly consistent and reliable

\section{DISADVANTAGES}

1. Reader collision

2. Tag collision 


\section{International Journal of Engineering Applied Sciences and Technology, 2020 Vol. 5, Issue 2, ISSN No. 2455-2143, Pages 439-444 \\ Published Online June 2020 in IJEAST (http://www.ijeast.com)}

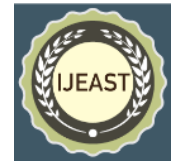

\section{CONCLUSION}

This project "Smart Cart Using RFID Technology" provides ease for shopping for the customers. It also consume less time when compared to regular billing technique. Customer need not want to wait in a long queue. Stock management can be monitored easily. Customer can purchase items with in their wallet balance as the total bill is displayed in the LCD display. This system is more reliable, also it doesn't require special training. This system reduce the number of salesmen. It accomplishes both customers and shopkeeper demand.

\section{ACKNOWLEDGEMENT}

We would like to thank our guide and professors, Computer Science and Engineering department, Mar Baselios Institute of Technology for their guidance and support. We find great pleasure in expressing our deep sense of gratitude towards all those who help us to complete this project successfully.

\section{REFERENCE}

[1] Arun Kumar, Rahul Hivare(2017), Smart shopping cart and stock management system - Hardware implementation and design of cart, (page 322)

[2] Ms. Rupali Sawat, Kripa Krishna, Shweta Bhokre, Priyanka Bhosale(2015), The RFID based smart shopping cart - Problem formulation, (page 279)

[3] T. Shanmugapriyan(2013), Smart cart to recogonize objects based on user intention - Smart cart (page 20502051)

[4] P.T Sivagurunathan, P Seema, M. Salini, R. Sindha(2018), Smart shopping trolley using RFID - The proposed work, (page 3783-3784)

[5] Ankush Yewatkar, Fai, Raj Singh, Ayushya, Amal Bhandal(2016), Smart cart with automatic billing, Product information, product recommendation using RFID and ZigBee with Anti-theft - motivation(page 794-795)

[6] Ruinan Li, Tianyi song, Nicolas Caparso, Jiguo Yu, Jason Coature, Xiuzhen Cheng(2017), IoT applications on Secure Smart Shopping system - mart shopping system(page 3-4)

[7] Akshay Kumar, Abhinav Gupta, S Balamurugan, S Balaji, Marimuthu R(2017), Smart shopping cart Methodolgy(page 2-3)

[8] Dr. Surya Prasad J, Praveen Kumar B.O, Roopan, Arjun A.K,(2011), A Novel low-cost Intelligent Shopping cart NLIC system Design(page 1-3)

[9] Vishwanadha V, Pavan Kumar P and Chirenjeevi
Reddy(2018), Smart shopping cart - Advantages and applications,(page 4)

[10] Manikandan T, Mohammed Aejaz, Nithin Krishna, N.M Mohan Kumar A.P, Manigandan R(2017), RFID based advanced shopping trolley for super market - proposed methodology(page 3-5)

[11] Ghatol Sonali Digambar, Mrs. V S Jahagidar, Mrs Pratiksha Dattacharya Khamitkar(2018), Smart shopping using Smart Trolley - Hardware reference(page 2-3)

[12] Rajukumar, K Gopalakrishna, K Ramesha(2013), Intelligent shopping cart - Result and Performance analysis(page 6-7)

[13] Dr. Sheifali Gupta, Ashmeet Kaur, Avni Garg, Abhishek Verma, Akshay Bansal, Arvinder Singh(2013) Adruino based smart cart - system Architecture(page 2-3)

[14] Ekta Maini, Jyoti Shettar(2014), Wireless intelligent trolley for malls - proposed system.

[15] Nemalidinne Sai Megana(2018), Design and implementation of a smart shopping cart by RFID technology - methodology(page $21-48$ ) 